Revista de Psicología Vol. 32 (1), 2014 (ISSN 0254-9247)

\title{
Síndrome de Munchausen by proxy: definición, contextualización y factores psíquicos involucrados
}

\author{
Thomás Gomes Gonçalves ${ }^{1}$, Maria Eduarda Germano Motta ${ }^{2}$, Paula Kegler ${ }^{3}$ \\ y Mônica Medeiros Kother Macedo ${ }^{4}$ \\ Pontificia Universidade Católica do Rio Grande do Sul-Brasil
}

\begin{abstract}
El Síndrome de Munchausen by proxy se refiere a una patología caracterizada por el abuso físico o emocional, en donde la simulación o producción de síntomas es direccionada al hijo, llevando a tratamientos de salud y cirugías innecesarios. Las dificultades diagnósticas de este tipo de abuso y los aspectos emocionales implicados llaman atención por los efectos destructivos en la subjetividad infantil, fruto de una falla en la capacidad de amar, proteger y priorizar las demandas del hijo. El Psicoanálisis ofrece una mirada que retrata un intento de elaboración de los propios conflictos psíquicos por la vía de la repetición de vivencias traumáticas. Así, se percibe la necesidad de una comprensión de la destrucción pulsional presente en la dinámica inconsciente materna revelada por esta modalidad de cuidado que se traduce en violencia.
\end{abstract}

Palabras clave: Síndrome de Munchausen by proxy, violencia infantil, Psicoanálisis

\section{Munchausen Syndrome by proxy: Definition, context, and psychological factors involved}

The Munchausen syndrome by proxy refers to a pathology characterized by physical and emotional abuse in which the simulation or production of symptoms is directed towards a child, which involves taking him or her to health treatments and unnecessary surgeries. The difficulties in the diagnosis of this form of abuse and the emotional aspects involved highlight the destructive effects in the infancy subjectivity due to the lack of loving capacity

1 Magister en Psicología por la Pontifícia Universidade Católica do Rio Grande do Sul. Dirección postal: Avenida Venâncio Aires 509/303, Porto Alegre-RS, 90040-193, Brasil. Contacto: gomes.thomas@gmail.com

2 Licenciada en Psicología por la Pontifícia Universidade Católica do Rio Grande do Sul (PUCRS). Contacto: mariaeduardagmotta@gmail.com

3 Magister en Psicología por la Pontifícia Universidade Católica do Rio Grande do Sul (PUCRS). Contacto: paulakegler@yahoo.com.br

4 Doctora en Psicología (Ph.D), Profesora de la Pontifícia Universidade Católica do Rio Grande do Sul (PUCRS). Contacto: monicakm@pucres.br 
protecting and prioritizing the child demands. Psychoanalysis offers a differentiated view, comprehending that the mother attempts to elaborate her own psychic conflicts by the repetition of traumatic experience. There is a necessity of comprehension of the instinct destructivity presented in the maternal unconscious dynamics revealed in the modality of caring which is reflected in violence.

Keywords: Munchausen syndrome by proxy, childhood violence, Psychoanalysis 


\section{Historial y caracterización del Síndrome de Munchausen}

La primera reseńa del Síndrome de Munchausen fue elaborada por el médico británico Richard Asher en 1951, quien la describió como una enfermedad en la cual los pacientes relatan historias con síntomas facticios, que conlleva a distintas investigaciones diagnósticas (Braga, 2007; Brito, Ramos, López \& Roca, 2000; Flaherty \& MacMillan, 2013; Forsyth, 1995; Mash et al., 2011; Meadow, 1995; Pankratz, 2010; Squires \& Squires, 2010). El síndrome obtuvo su nombre debido a una asociación con la historia de Karl Friedrich Hieronymus, el Barón de Münchhausen (1720-1797), conocido por ser el "Barón Mentiroso", ya que contaba aventuras increíbles sobre las batallas vividas en la guerra (Braga, 2007). En este sentido, este síndrome se caracteriza por los traslados de los enfermos entre distintas instituciones sin que se resuelvan sus problemas de salud (Brito et al, 2000). De este modo, los "pacientes son también peregrinos, viajando de hospital en hospital, contando sus historias fantásticas sobre enfermedades e inventando síntomas con los que captan la atención de los médicos, exhibiendo el arte como verdaderos ilusionistas" (Penna, 1992, p.341).

Una modalidad de este cuadro patológico es el Síndrome de Munchausen by proxy (SMP), denominado también Síndrome de Munchausen by proxy o por terceros. De esta manera, el término by proxy refiere a que la persona afectada por la enfermedad le induce los síntomas a otra (Yorker \& Kahan, 1990; Flaherty \& MacMillan, 2013; Squires \& Squires, 2010). Dicha caracterización ya fue nombrada como Sindrome de Polle, en alusión al único hijo del Barón de Münchausen cuya muerte, acontecida en el primer año de edad, posiblemente fue causada por su propio padre. Por ello, el SMP se define como un trastorno facticio, en donde se induce la simulación o creación de una enfermedad física o psicológica a un tercero, el cual es por lo general 
el(la) hijo(a), quien es llevado al hospital repetidas veces (Braga, 2007). Dicha forma de maltrato físico o emocional puede acarrear graves secuelas o, incluso, la muerte del niño.

Según Meadow (2002), el Síndrome de Munchausen by proxy se puede utilizar para describir formas específicas de maltrato infantil, o para dar nombre al diagnóstico atribuido al perpetrador, quien en la mayoría de los casos es la madre. El autor resalta que muchos casos de SMP son diagnosticados tarde, por lo que hay niños que realmente fueron víctimas de SMP, pero cuya causa de óbito fue atribuida al Síndrome de la Muerte Repentina infantil. De esta forma, se observa la evolución patológica y abusiva que caracteriza al SMP, el cual, según Braga (2007), acomete a padres o responsables que provocan enfermedades imaginarias o reales en sus hijos, perjudicando su salud a través de tratamientos y cirugías innecesarias.

Trajber y colaboradores (1996) señalan que el comportamiento de la madre perpetradora simula sentimientos de preocupación y devoción por el hijo, lo que dificulta el diagnóstico. A su vez Mehl, Coble y Johnson (1990) afirman que hay casos en donde el niño participa simbióticamente de esta situación.

En relación a las características de los niños víctimas de este síndrome, Beard (2007) afirma que son, por lo general, individuos con menos de dos años de edad, siendo raros los casos de niños con 6 o más años. El autor afirma que muchos de ellos colaboran con los padres en la creación de sus síntomas para ganar algún tipo de aceptación. Sobre los perpetradores, Fraser (2008) estima que entre el 90\% y 98\% son mujeres, y que las madres constituyen el $85 \%$ del total de los abusadores. Otros perpetradores del género femenino pueden ser enfermeras o niñeras.

En este sentido, Bartsch, Ribe, Schutz, Weigand y Weiler (2003) relatan que más de 700 casos fueron descritos en la literatura científica específica alrededor del mundo como una forma radical de maltrato con alta mortalidad. Asimismo, se presentan indicios de que, cuando un nińo es víctima del Síndrome de Munchausen by proxy, posiblemente sus hermanos sufrirán los mismos tipos de maltrato si la 
patología no es diagnosticada a tiempo. Los autores también aseguran que el diagnóstico de este síndrome puede demorar años, debido a la gran disimulación del comportamiento materno.

De igual forma, en el SMP los padres usan distintos ardides para provocar la enfermedad de los hijos y obtener ventajas secundarias durante el internamiento. Para ello, suelen contar historias falsas con respecto a la dolencia, distorsionando síntomas e induciendo indicios inexistentes y de difícil diagnóstico (Brito et al., 2000; Flaherty \& MacMillan, 2013; Mash et al., 2011; Squires \& Squires, 2010). Braga (2007) refiere que el perpetrador simula o crea enfermedades en el(la) hijo(a) con el fin de recibir atención médica. De esta manera, Barrios, Couse y Corral (1996) consideran el SMP como un modo de agresión denominado maltrato intencional. A esto se suma la concepción de Meadow $(1982 ; 1993 ; 2002)$ que entiende tal síndrome como una "perversión de parentalidad", en el cual se encuentra una falla en la capacidad de amar, proteger y dar prioridad a las demandas de los hijos por encima de las propias necesidades.

En el Manual Diagnóstico y Estadístico de Trastornos Mentales - DSM-IV-TR (APA, 2002) el SMP está relacionado con los Trastornos Facticios, con la siguiente descripción diagnóstica: creación o simulación intencional de indicios o síntomas físicos o psicológicos; la motivación para el comportamiento consiste en asumir el papel de enfermo; ausencia de incentivos externos para el comportamiento, tales como ventajas económicas. Además, los Trastornos Facticios son codificados en base al predominio de indicios y síntomas psicológicos, físicos o ambos.

Los criterios de diagnóstico para casos de SMP adoptados por Meadow (2002) consideran la presencia de los siguientes factores: (a) existencia de enfermedad producida, ya sea falsa o inducida por los padres o por alguien que vive con el niño; (b) el niño es llevado al hospital repetidas veces y el perpetrador niega que sea el causador de la enfermedad; (c) el niño mejora cada vez que se lo separa de su perpetrador y este trata de asumir una posición de enfermo. Bartsch y colaboradores (2003) usan estas circunstancias para mostrar la 
presencia de SMP: reiterados síntomas de enfermedad, repetidas visitas al hospital o al médico, múltiples procedimientos sin que se establezca un diagnóstico exacto, resistencia a la terapia, aparición de enfermedad o muerte de hermanos, detección única o múltiple de sustancias inusuales en tejidos de órganos preservados y detección histológica de reiterados intentos de asfixia.

La distinción teórica entre simulación, Síndrome de Munchausen e histeria realizada por Eisendrath (1984) llevan a Brito y colaboradores (2000) a sugerir una relectura y adaptación de dicha distinción diagnóstica. En la histeria, los autores consideran que, tanto la creación de los síntomas de la enfermedad como su motivación son mecanismos inconscientes. Por otro lado, refieren que en el Síndrome de Munchausen ocurre una simulación consciente. Conforme a esto, en las enfermedades ficticias, como es el caso del SMP, la producción de los síntomas es intencional, sin embargo, las personas están inconscientemente impedidas de entender los motivos que producen dichos síntomas (Brito et al., 2000; Del Casale et al., 2012).

Al tratar de abordar las causas de este síndrome, Meadow (2002) señala que es común encontrar un historial de trastorno facticio en la vida de las madres maltratadoras. Respecto a los padres abusivos, la investigación realizada por Meadow (1998) demostró que 15 tuvieron algún tipo de trastorno somático y 5 un evidente diagnóstico de Síndrome de Munchausen. Ello comprueba la necesidad de entender aspectos familiares más amplios involucrados en la configuración del SMP. Esto debido a que se aprecia que la salud psíquica del adulto perpetrador está comprometida por motivos que todavía son superficialmente comprendidos por los estudios realizados sobre este tema.

\section{Aportes contextuales acerca del Síndrome de Munchausen by proxy}

Si se tiene en cuenta que el Síndrome de Munchausen by proxy es más común en las madres (Del Casale et al., 2012; Morais et al., 2006; Trajber et al., 1996), se observará que la repercusión de las 
enfermedades ficticias sobrepasa el dúo madre-hijo, ya que todo el contexto familiar, social y económico es incalculablemente afectado (Braga, 2007; Brito et at., 2000). En gran parte de los casos el cónyuge se encuentra física o emocionalmente ausente (Del Casale et al., 2012; Morais et al., 2006). A su vez, Braga (2007) considera que la relación conyugal insatisfactoria puede estar relacionada con un sentimiento de rechazo ya vivenciado por la madre en su propia infancia.

En la mayoría de los casos, las madres perpetradoras son mujeres entre 20 y 30 años (Morais et at., 2006). Moreira y Moreira (1999) señalan que estas madres suelen ser calmadas, demuestran confiar en los médicos, y necesitan obtener su atención. Además, Forsyth (1995) indica que la relación establecida por la madre con el niño se caracteriza por ser excesiva. Por lo general, las víctimas del SMP son niños de edad preescolar; no obstante, es posible encontrarlo en recién nacidos, adolescentes e incluso adultos (Braga, 2007; Del Casale et al., 2012; Flaherty \& MacMillan, 2013; Squires \& Squires, 2010). Una evaluación psicológica del caso clínico presentado por Morais y colaboradores (2006) señaló que la madre se esforzaba tratando de demostrar orden y estabilidad en la estructura de la familia. Sin embargo, durante el acompañamiento se constataron evidentes alteraciones en la dinámica familiar.

Braga (2007) afirma que para simular la enfermedad de los hijos, los padres perpetradores llevan a cabo acciones tales como: adulteración de muestras para análisis clínicos, cambio muestras, uso excesivo de laxantes, cambio de remedios manteniendo el envase original, asfixia con parada cardiorrespiratoria y alteración de los datos del termómetro. Una investigación realizada en Inglaterra por Maida, Molina y Carrasco (1999) usó filmaciones en 39 casos investigados por sospecha de SMP. En 33 casos, los padres o cuidadores asfixiaron a los niños, les dieron sustancias tóxicas, provocaron fracturas o les golpearon durante la internación.

Un caso clínico relatado por Trajber y colaboradores (1996) sobre el SMP permitió percibir el comportamiento de la madre durante el período en que su hija estuvo internada. Se subrayó su soltura en la relación con los médicos y el equipo de enfermería, la agresiva hostilidad 
con el cónyuge y, sobre todo, su entusiasmo por difundir el caso, que fue noticia en la prensa. Los autores creen que el deseo de obtener notoriedad por medio de periódicos y televisión refuerza la satisfacción de sus propias necesidades al usar al niño de forma abusiva. La evaluación psiquiátrica de la madre, en el caso relatado por Trajber y colaboradores (1996), comprobó la presencia de padecimiento psíquico intenso e importante perjuicio de la autoestima.

A su vez, se subraya que muchas de estas madres maltratadoras tienen formación profesional o conocimientos sobre sanidad, y tienden a sentirse cómodas en el ambiente hospitalario. Por lo tanto, suelen aprobar la realización de análisis, demostrando inclusive calma y frialdad ante el sufrimiento de los nińos (Flaherty \& MacMillan, 2013; Morais et al., 2006; Squires \& Squires, 2010). Scheirer (2000) afirma que la mayor dificultad vinculada al diagnóstico de SMP es detectar la enfermedad pues, por lo general, el perpetrador presenta al hijo con sintomatología similar a las descripciones encontradas en libros científicos. De esta manera, parece que el perpetrador usa intencionadamente un conocimiento previo sobre determinada enfermedad para inducirle al hijo los síntomas correspondientes y, de este modo, lograr convencer al equipo sanitario sobre la veracidad de la patología relatada.

Los patrones de simulación que comúnmente se relatan son los del tipo abdominal agudo, caracterizados por dolores abdominales e historial de varias cirugías; o del tipo hemorrágico y neurológico, cuya principal característica son quejas por cefaleas, pérdida de conocimiento o convulsiones (Asher, 1951; Braga, 2007; Talbot, 1992). Asimismo, el envenenamiento es la forma de provocar enfermedad más descrita en la literatura médica; en estos casos, el niño es inicialmente sometido a la ingestión de pequeñas cantidades en casa. Los relatos señalan que las dosis aumentan gradualmente hasta su internación (Meadow, 1993; Pires \& Molle, 1999).

Trajber y colaboradores (1996) se refieren a otra forma típica de manifestación del SMP, cuando relatan que los cuadros clínicos del síndrome por lo general incluyen episodios de hemorragia gastrointestinal, urinaria y de las vías aéreas superiores. Estos síntomas pueden 
ser simulados cuando la madre mezcla su sangre en el cuerpo del niño, o se producen cuando ella le inyecta algo. Además de las hemorragias, Trajber y colaboradores (1996) señalan anormalidades neurológicas y convulsiones, fiebres, apnea, vómitos, diarrea e hipoglicemia como indicios comúnmente presentados.

Los síntomas físicos son más comunes en los niños, aunque los efectos psíquicos también surjan y exijan tratamientos e interacciones. Dichos síntomas tienen relación con la presencia de trastornos de estrés postraumático, disturbios de atención, problemas de interacción social, baja autoestima, inmadurez, pasividad, relaciones anormales con las madres y comportamiento agresivo (Braga, 2007). En un estudio llevado a cabo por Forsyth (1995), se observaron casos de trastornos alimentarios en niños de hasta tres ańos de edad, y comportamiento retraído o hiperactivo en preescolares.

Una investigación realizada en Inglaterra e Irlanda por McClure, Davis, Meadow y Sibert (1996) durante un período de dos años tuvo el propósito de identificar cuántos casos de Síndrome de Munchausen by proxy tenían lugar por año en los dos países, en instituciones hospitalarias que atendían niños y adolescentes de hasta 16 ańos. Los resultados mostraron que hubo 128 casos de asfixia y envenenamiento no accidental en ese período de tiempo. Se estima que en esos dos países hay una incidencia de 0.5 cada 100000 sujetos de hasta 16 años; un $77 \%$ de los casos fueron cometidos contra niños de menos de cinco años, y considerando apenas ese grupo, la prevalencia es de 1.2 cada 10000 niños. Sin embargo, al examinar a los nińos de hasta un ańo de edad, esa incidencia es de 2.8 por cada 100000 nińos (McClure et al., 1996). Dichos datos sugieren que el SMP es más frecuente en niños de menor edad, y posiblemente más indefensos. Con relación al género, el estudio señaló que un $47 \%$ de las víctimas eran del sexo masculino y un 53\% del sexo femenino, sin que hubiera una diferencia estadísticamente significativa. La madre fue identificada como la que cometió los abusos en 109 casos, representando un 85\% del total de perpetradores. Asimismo, ocho niños murieron como resultado de los maltratos recibidos. 


\section{Relevancia del estudio y de la difusión del Síndrome de Munchausen by proxy}

Es importante destacar que en las últimas décadas se ha presentado una creciente medicalización de la sociedad, sobre todo debido al importante desarrollo y avance de la tecnología aplicada a la medicina. Por ello, las instituciones sanitarias atienden a muchos pacientes con enfermedades más complejas y que demandan recursos para diagnosticar sus quejas y padecimientos (Brito et al., 2000; Morrell \& Tilley, 2012).

En este sentido, el Síndrome de Munchausen by proxy puede ser considerado una patología de alta complejidad, poco conocida y poco diagnosticada en Brasil. Feldman y Brown (2002) constataron, tras una revisión de la literatura, que la mayor parte de los trabajos científicos sobre el SMP son publicados en países de habla inglesa. A su vez, Braga (2002) subraya que es necesario estudiarlo con el fin de que los equipos sanitarios estén capacitados para distinguirlo y diagnosticarlo, evitando más daños a la víctima. El autor resalta la importancia de que esta temática sea investigada en el ámbito nacional con vistas a facilitar el abordaje ante tanta violencia con los nińos.

Por ello, es fundamental para el tratamiento que los equipos reconozcan la enfermedad, abarcando las esferas públicas y privadas de la sanidad, así como la asistencia social y jurídica (Braga, 2007; Morrell \& Tilley, 2012). Forsyth (1995) considera que se podrán diagnosticar más casos del Síndrome de Munchausen by proxy en la medida que los profesionales sean conscientes de su existencia. Un grupo multidisciplinario compuesto por pediatras, psiquiatras, psicólogos, asistentes sociales y abogados debe estar capacitado para el manejo y evaluación de estos casos clínicos (Farinatti, Biazus \& Leite, 1993; Pires \& Molle, 1999).

Entretanto, el equipo sanitario puede, inclusive sin querer, reforzar o aceptar la violencia con el objetivo de obtener rápidamente un diagnóstico, e indicar un tratamiento a partir de la manifestación sintomatológica (Awadallah, Vaughan, Franco, Sharaby \& Goldfarb, 2005). Meadow (1982) señala algunas conductas cuyo fin es facilitar 
el reconocimiento del síndrome ante casos clínicos, precisando que es necesario comprobar la relación temporal de los síntomas con la presencia de la madre, separándola del niño para observar el cuadro. Del mismo modo, es imprescindible verificar la veracidad de los indicios, guardando el material para investigarlo.

Por ello, es indispensable que los equipos sanitarios presten atención cuando un niño en tratamiento presenta síntomas clínicos que no corresponden con lo esperado tras una intervención terapéutica. De esta forma, síntomas sin sentido, internaciones frecuentes, y realización de pruebas diagnósticas con resultados negativos, son muestras que ayudan a reconocer el síndrome (Morais et al., 2006; Morrell \& Tilley, 2012). Por lo general, los tratamientos son vistos como ineficaces y los padecimientos son inexplicables, raros y de larga duración, mientras que los síntomas se manifiestan sólo cuando la madre está cerca (Brito et al., 2000; Pankratz, 2010; Squires \& Squires, 2010).

En este sentido, Trajber y colaboradores (1996) afirman que es necesario estar atentos a los indicios que aparecen en los nińos cuando la madre está cerca ya que, aunque la madre esté presente durante la internación y procure que los síntomas sean investigados, no parece estar muy preocupada con la gravedad del caso. Braga (2007) considera que la ruptura de la relación destructiva entre la madre y el niño es una dificultad encontrada por los profesionales del equipo sanitario, puesto que la relación patológica se caracteriza por la intensa simbiosis entre ambos.

Debido a lo expuesto, se discute si es apropiada una propuesta de prevención para los casos de SMP. Eminson y Jureidini (2003) sugieren que para lograr una verdadera acción preventiva es importante que la intervención primero se realice con los profesionales del área médica. A su vez, Trajber y colaboradores (1996) destacan que, debido a los factores emocionales involucrados en el síndrome, la realización de una anamnesis psicosocial y la ayuda psiquiátrica y psicológica son aspectos importantes para prever el diagnóstico. No obstante, para modificar las prácticas médicas es imprescindible intentar comprender los aspectos psíquicos involucrados en la actitud del perpetrador. 


\section{Factores psíquicos involucrados en el SMP: contribuciones del psicoanálisis}

El Psicoanálisis ofrece una propuesta consistente sobre los complejos elementos psíquicos propios del ser humano, ofreciendo recursos que permiten una amplia investigación de los cuadros psicopatológicos, y presentando herramientas que proporcionan condiciones de lectura y comprensión de lo que está más allá de la racionalidad y de la consciencia. De este modo, las complejidades psíquicas involucradas en el fenómeno del SMP pueden ser entendidas a la luz de la teoría psicoanalítica.

Estudios realizados sobre el SMP indican que la peregrinación por hospitales, al igual que la realización de análisis y tratamientos innecesarios en el niño, por lo general se revierte en beneficio de la madre (Fisher \& Mitchell, 1995; Meadow, 1995: Morley, 1995; Pankratz, 2010; Squires \& Squires, 2010). Se puede pensar que la advertencia de los autores con relación a la actitud materna manifiesta un intento de elaborar sus propios conflictos psíquicos a través de la repetición de los procedimientos de investigación e intervención médica impuestos al niño.

Los aportes teóricos del Psicoanálisis ofrecen una pertinente comprensión acerca del fenómeno de la repetición, al discernirlo como una compulsión de origen inconsciente, por medio de la cual "el sujeto se coloca activamente en situaciones penosas, repitiendo así experiencias antiguas" (Laplanche \& Pontalis, 2001, p. 83). Esta condición puede ser considerada como una manifestación psíquica del predominio de la pulsión de muerte propuesta por Freud (1920/1996) en el texto Más allá del principio del placer.

Siguiendo esta línea, la teoría freudiana señala que dificultades o incapacidades de representaciones psíquicas, resultado de experiencias traumáticas, pueden causar muerte en el espacio psíquico. De este modo, Freud (1920/1996) inaugura una mirada con respecto a las manifestaciones del sujeto psíquico que expresan la existencia de fuerzas destructivas y disyuntivas, y cuyas manifestaciones en acto cuentan la intensidad de las experiencias que no pueden tener un sentido atribuido 
por la protagonista. La pulsión de muerte, según la propuesta del Psicoanálisis, sirve para una función de desconexión, de inmovilidad, de búsqueda de la reducción completa de las tensiones. Los conceptos de pulsión de muerte y de compulsión a la repetición se aplican a medida que estos fenómenos psíquicos sirven al aparato psíquico para intentar dominar un exceso de excitaciones que podría estar acompańado de un sentimiento de desagrado. Una vivencia colocada en acto que comunica una falla en la capacidad de representación remite al fracaso del universo simbólico proveniente de "un funcionamiento arcaico y con alternativas limitadas de salida de energía" (Gobbi, 2005, p.143).

En este sentido, el comportamiento de la madre perpetradora en el SMP revela la destructividad pulsional presente en su dinámica y su economía psíquica. La distorsión de la realidad presente en la sintomatología forjada por la madre remite a su historia, de modo que se puede considerar que en los delitos de la madre se manifiesta la reproducción de experiencias traumáticas. Esto implica que, a través de la repetición de estas situaciones impuestas al hijo, la perpetradora procura reproducir activamente algo que sufrió pasivamente, siendo el acto ejecutado por ella una tentativa de dominar experiencias desagradables originadas en su propia historia.

La comprensión de la perversión de la parentalidad propuesta por Meadow (2002), con el fin de encontrar un sentido para el abuso materno del SMP, indica una carencia en los cuidados relacionados con el amor y protección ofrecidos al hijo. Conforme a esto, se cuestionan las condiciones del cuidado ofrecido a estas mujeres en sus historias de vida, cuya hipótesis de precariedad o inexistencia parece confirmada en la actual repetición compulsiva de su comportamiento como madre, denunciando un perjuicio en la modalidad de cuidado que se traduce en violencia.

Jureidini (1993) trae importantes colaboraciones para comprender el SMP al afirmar que, en casos ficticios de complicaciones obstétricas de madres con historial de haber abusado de sus hijos en el SMP, hubo una transición de comportamiento hostil autoinfligido hacia un comportamiento hostil externo, dirigido hacia el niño. De esta manera, 
llama la atención la nueva dirección de la hostilidad de esas mujeres, constituida a partir del nacimiento de un hijo.

La hostilidad autodirigida y posteriormente canalizada hacia el exterior, presente en el SMP, conduce también a un comportamiento resultado de la preponderancia de la pulsión de muerte. Laplanche y Pontalis (2001) señalan que "inicialmente volcadas hacia el interior y tendiendo a la autodestrucción, las pulsiones de muerte después serían dirigidas al exterior, manifestándose entonces bajo la forma de pulsión de agresión o de destrucción" (p. 407). La sintomatología materna resultado de una historia de vida dolorosa - coloca en escena una trama psíquica permeada por excesos traumáticos. La postura de una madre y cuidadora que es, al mismo tiempo, abusiva y dominadora, revela los aspectos destructivos de su funcionamiento psíquico. De este modo, las manifestaciones de crueldad presentes en el SMP demuestran el potencial mortal de la repetición y de la pulsión de muerte, y demandan una mirada más allá de las manifestaciones evidentes de la conducta materna.

\section{Consideraciones finales}

Es importante destacar que la mayoría de los estudios y publicaciones sobre este fenómeno se encuentran en revistas de pediatría. Por ello, es necesario incluir esta temática en la discusión científica del área de Psicología, debido a las implicaciones emocionales de la madre y del nińo, sobre todo en lo que se refiere a la salud psíquica del nińo, y las condiciones de desamparo y dependencia que caracterizan el proceso de constitución de la subjetividad infantil.

Se sabe que las relaciones iniciales que un niño establece con sus cuidadores son, de cierta forma, definitorias de la salud psíquica del individuo. Por lo tanto, los aportes del Psicoanálisis contribuyen con el entendimiento inherente a la relevancia que adquieren los factores psíquicos en la constitución de un sujeto. De este modo, al considerarse los factores inconscientes referentes a ese fenómeno, se abre una posibilidad de comprender en profundidad los factores que estén promoviendo esta conducta materna marcada por la violencia. 
Es imprescindible promover una mayor consciencia de los profesionales sanitarios sobre el potencial mortal, tanto desde el punto de vista físico como psíquico de la conducta de la madre con relación al niño cuando se sospecha o se confirma el SMP. Asimismo, es relevante que se fomente la realización de investigaciones a través de las cuales se pueda acceder al discurso de estas madres y no apenas a los datos estadísticos referentes a la frecuencia del fenómeno. La identificación de factores psíquicos propios de este contexto intersubjetivo marcado por la violencia podrá contribuir significativamente para comprender mejor el fenómeno al desvelar factores que no son identificados cuando el SMP es investigado por sus aspectos más visibles y cuantificables.

\section{Referencias}

APA - American Psychiatric Association. (2002). Manual Diagnóstico dos Transtornos Mentais. DSM IV-TR, $4^{\mathrm{a}}$ ed. Porto Alegre: Artmed. Asher, R. (1951). Munchausen's syndrome. Lancet, 1, 339-341. Awadallah, N., Vaughan, A., Franco, K., Sharaby, N. \& Goldfarb, J. (2005). Munchausen by proxy: A case, chart series, and literature review of older victims. Child Abuse \& Neglect, 29(8), 931-941. Barrios, A. I. C., Couse, G. F. \& Corral, J. P. (1996). Niño maltratado. Revista Cubana de Pediatria, 68(3), 1996.

Bartsch, C., Ribe, M., Schutz, H., Weigand, N. \& Weiler, G. (2003). Munchausen syndrome by proxy (SMP): an extreme form of child abuse with a special forensic challenge. Forensic Science International, 137, 147-151.

Beard, K. (2007). Protect the children: be on the lookout for Munchausen Syndrome by Proxy. RN Journal, 70, 33-37.

Braga, M. S. (2007). Um estudo teórico sobre a Sindrome de Münchausen por Procuração. Manuscrito não publicado.

Brito, A. D. E., Ramos, B. F. R., López, J. B. M. \& Roca, A. A. E. (2000). Síndrome de Munchausen: Un reto para el clínico. Revista Cubana de Medicina, 39(4), 228-237. 
Del Casale, A., Ferracuti, S., Rapinesi, C., Serata, D., Simonetti, A., Caloro, M.,..., Girardi P. (2012). Factitious disorder comorbid with Bipolar I disorder. A case report. Forensic Science International, 219, 37-40.

Eisendrath, S. J. (1984). Factitious illness: a clarification. Psychosomatics, 25(2), 110-3, 116-7.

Eminson, M. \& Jureidini, J. (2003). Concerns about research and prevention strategies in Munchausen syndrome by proxy (SMP) abuse. Child Abuse \& Neglect, 27(4), 413-420.

Farinatti, F., Biazus, D. B. \& Leite, M. B. (1993). Pediatria social: a criança maltratada. Rio de Janeiro: Medsi.

Feldman, M. D. \& Brown, R. M. A. (2002). Munchausen by proxy in an international context. Child Abuse \& Neglet, 26(5), 509-524.

Fisher, G. C. \& Mitchell, I. (1995). Is Munchausen syndrome by proxy really a syndrome? Archives of disease in childhood, 72(6), 530-534.

Flaherty, E. \& MacMillan, H. (2013 Sep). Caregiver-Fabricated Illness in a Child: A Manifestation of Child Maltreatment. American Academy of Pediatrics, 132 (3), 590-597. doi:10.1542/ peds.2013-2045

Fraser, G. (2008). A mother's investment in maintaining illness in her child: A perversion of mothering and of women's role of 'caring'? Journal of Social Work Practice, 22, 169-180.

Forsyth, B. W. C. (1995). Síndrome de Münchausen por procuração. En L. Melvin (Org.), Tratado de psiquiatria da infância $e$ adolescência (pp. 1042-1049). Porto Alegre: Artes Médicas.

Freud, S. (1920/1996). Além do princípio do prazer. En J. Strachey (Ed. y Trad.), Edição Standard das Obras Psicológicas Completas de Sigmund Freud (Vol.18, pp. 17-75). Rio de Janeiro: Imago.

Gobbi, A. S. (2005). De quê padece o sujeito na atualidade? Revista da Sociedade de Psicologia do Rio Grande do Sul, 4(1), 139-152.

Jureidini, J. (1993). Obstetric factitious disorder and Munchausen Syndrome by Proxy. Journal of Nervous and Mental Diseases, 182(2), 135-139. 
Laplanche, J. \& Pontalis, J.B. (2001). Vocabulário da psicanálise. São Paulo: Martins Fontes.

Maida, A. M., Molina, M. E. \& Carrasco, X. (1999). Síndrome de Münchausen-por-poder: Um diagnóstico a considerar. Revista Chilena de Pediatria, 70(3).

Mash, C., Frazier, T., Nowacki, A., Worley, S. \& Goldfarb, J. (2011). Development of a Risk-Stratification Tool for Medical Child Abuse in Failure to Thrive. Pediatrics, 128 (6)1467-1473.

McClure, R. J., Davis, P. M., Meadow, S. R. \& Sibert, J. R. (1996). Epidemiology of Munchausen syndrome by proxy, nonaccidental poisoning, and non-accidental suffocation. Archives of Disease in Childhood, 75, 57-61.

Meadow, R. (1982). Munchausen syndrome by proxy. Archives of disease in childhood, 57(2), 92-98.

Meadow, R. (1993). False allegations of abuse and Munchausen syndrome by proxy. Archives of disease in childhood, 68, 444-447. Meadow, R. (1995). What is, and what is not, 'Munchausen syndrome by proxy'?. Archives of disease in childhood, 72, 534-538.

Meadow, R. (1998). Munchausen syndrome by proxy abuse perpetrated by men. Archives of disease in childhood, 78, 210-216.

Meadow, R. (2002). Different interpretations of Munchausen Syndrome by proxy. Child Abuse \& Neglect, 26(5), 501-508.

Mehl, A. L., Coble, L. \& Johnson, S. (1990). Munchausen Syndrome by proxy: A family affair. Child abuse \& Neglect, 14, 577-585.

Morais, M. B., Marchette, A., Speridião, P., Lopes, L., Robles, R., Benedito, M. \& Neto, U. (2006). Síndrome de Munchausen por terceiro simulada como alergia alimentar múltipla: relato de caso. Revista Paul Pediatria, 24(4), 373-378.

Moreira, E. C. \& Moreira, L. A. C. (1999). Hipocondria por procuração: Relato de dois casos. Jornal de Pediatria, 75(5), 373-376.

Morley, C. J. (1995). Practical concerns about the diagnosis of Munchausen syndrome by proxy. Archives of disease in childhood, 72(6), 528-538. 
Morrell, B. \& Tilley, D. (2012). The role of Nonperpetrating Fathers in Munchausen Syndrome by Proxy: A Review of the Literature. Journal of Pediatrics Nursing, 27, 328-335.

Pankratz, L. (2010). Persistent problems with the "separation test" in Munchausen syndrome by proxy. The Journal of Psychiatry \& Law, 38, 307-323.

Penna, T. L. M. (1992). Desordens fictícias. En J. M. Filho (Org.), Psicossomática hoje (pp. 340-343). Porto Alegre: Artes Médicas.

Pires, J. M. A. \& Molle, L. D. (1999). Síndrome de Münchausen por procuração - Relato de dois casos. Jornal de Pediatria, 75(4), 281-286.

Scheirer, H. A. (2000). Factitious disorders by proxy in wich the presenting problem is behavioral or psychiatric. Journal American Academy of Child and Adolescent Psychiatry, 39(5), 668-670.

Squires, J. \& Squires, Jr. (2010). Munchausen Syndrome by Proxy: Ongoing Clinical Challenge. Journal of Petroleum Geology, 51(3), 248-253.

Talbot, J. A. (1992). Tratado de psiquiatria. Porto Alegre: Artes Médicas. Trajber, Z., Murahovski, J., Candio, S., Cury, R., Gomide, C., Klein, E. \& Tofolo, V. (1996). Síndrome de Munchausen por procuraçáo: o caso da menina que sangrava pelo ouvido. Jornal de Pediatria, 72(1), 35-39.

Yorker, B. C. \& Kahan, B. B. (1990). Münchausen's syndrome by proxy as a form of child abuse. Archives of Psychiatric Nursing, 4(5), 313-318.

Recibido: 11 de marzo de 2013 Aceptado: 03 de diciembre de 2013 